\title{
Ethernet-based network with high reliability for railway signaling systems
}

\author{
H.-J. Jo, J.-G. Hwang \& Y.-K. Yoon \\ Train Control Research Team, Korea Railroad Research Institute \\ (KRRI), Korea
}

\begin{abstract}
Railway signaling systems are computerized equipment vital for the guarantee of the safe running of trains. Because of the computerization of railway signaling systems, the importance of a networking interface between these pieces of equipment is increasing. Thus, it is important to have a reliable and safe communication link between signaling equipment. Recently, network technologies have been applied in a real-time industrial control system, and also there are several studies applying computer network technology in vital control systems such as railway signaling systems. To deploy these computer network related studies in railway signaling systems, implementation costs, transmitted reliability, safety assurance technique and compatibility etc, are considered. The computer network protocol for railway signaling systems has an important advantage over the widely used Ethernet in terms of deterministic characteristics. In this paper, we propose computer network technology, especially the Ethernet, for railway signaling systems. The computer network characteristics of real-time industrial control systems and vital railway signaling systems are presented in this paper. This algorithm presented is based on switched Ethernet technology with a redundancy scheme. Also we demonstrate the experimental results of the proposed network algorithm.
\end{abstract}

\section{Introduction}

Railway signaling systems are computerized vital systems for the guarantee of the running trains safely which take charge of controlling train speed and direction, especially preventing train collisions. Every signaling system plays 
each role, and is linked with other signaling systems, which collectively form one big signaling system.

The links between controlling equipment for railway signaling have some problems with maintenance and repairs and on adding new equipment because the way of linking is point-to-point communication. Recently, railway advanced countries are trying to study and develop ways of linking signaling systems by networking them, and some of the countries have already used the network method $[1,2]$. The advent background of studying railway signaling system networks, using science technology, is an increasing need of networking for transmitting data between railway signaling systems.

Industrial network technologies were earnestly developed from the middle of the 1980s, and network technologies for Ethernet-based industrial control systems, such as RETHER, 3ComPACE, HP AnyVG LAN, IEEE 802.1p which are MAC class mechanism for supporting the real-time traffic, are studied. To apply industrial networks to railway signaling systems, choosing appropriate protocols should include considering various elements. On a broad view, cost, data transmission rate, extensibility, supporting media, the ways of media connection, and other elements should be considered. Considering these elements, the network of railway signaling systems is consequently determined to be developed by Ethernet-based protocol.

Ethernet appears as the next generation industrial network because of its open structure and low-cost. Recently, the switched Ethernet shows a very promising prospect for industrial networking because the switching technology can eliminate frame collisions. Ethernet applications to industrial controlling systems was impossible because the real-time requirements were not satisfied owing to the random characteristics of the Ethernet protocol, but the recently advanced switched Ethernet enables the Ethernet technology to be applied to industrial controlling systems [4]. By the theoretical explanations of the switched Ethernet, we identify the possibility of applying it to railway signaling systems.

In this paper, we analyze the performing efficiency through an Ethernet-based network algorithm which has high reliability, including the algorithms for detecting deficiencies and its recovery when network defects occurring. If the proposed Ethernet-based network algorithm is applied to interfaces between railway signaling systems, greater development of the extension and the maintenance and repairs of the signaling control systems can be expected, and so does the consistent operation of the signaling control systems, accompanied by a guarantee of the communication system's reliability.

\section{Mathematical analysis of Ethernet for railway signaling systems}

If these Ethernets are directly applied to the railway signaling systems, and transmitted data do not have regular transmission delays, then the Ethernets problematically have random transmission delays namely, uncertainty of transmission delays. And, the methods to reduce the number of collisions in Ethernet systems also have problems, because people should modify data link 
classes or TCP/IP classes directly. In this paper, one problem of the Ethernet applications is solved by bringing the switching technologies as one way to solve the uncertainty of transmission delay in the Ethernet. The switching technologies were mainly developed and applied to the office network, but their trials for applications to industrial networks are increasing, as their generalization and cost decrease.

\subsection{Ethernet and switched Ethernet}

IEEE 802.3, often referred to as Ethernet, was developed for data communications among computers in the early 1970s by the IEEE, and is the basis of a physical layer and a data link layer of the office communication. The CSMA/CD (Carrier Sensing \& Multiple Access/Collision Detection) is the media access control method of IEEE 802.3. In the Ethernet, the more there is of traffic by the CSMA/CD algorithm, the more collisions occur, so the transmission delays are increasing by waiting for the backoff time which are as long as the times of collisions. And also, the delaying time cannot be predicted because of the delaying by the random time following the BEB algorithm [4].

The switched Ethernet differs from the conventional one. In the switched Ethernet, collisions between stations are prevented because the dedicated virtual circuits between the stations, which communicate through switches, are installed. Collisions do not occur in thsee cases of which many stations transmit simultaneously because the switches send frames only to the determined destination stations when the source stations transmit frames. Also, collisions do not occur before the frames are not received because the full duplex method is used in the switched Ethernet, which performs by sending and receiving by each line.

A typical method of switching technology is the store and forward method. The switch receives a frame through a transmission line from a source station, and then checks if the reception line of a destination station is idle. If the reception line is idle, the switch transmits the frame. Otherwise, the switch stores the frame into its buffer and waits until the reception line becomes idle. In addition, if several frames with the same destination address are received at the switch simultaneously, the switch stores frames to the buffer and then sends frames to the destination one by one.

\subsection{Theoretical analysis of maximum transmission delay for Ethernet and switched Ethernet}

The performance analysis of Ethernet has not yet been exactly achieved because of the probability characteristics of the BEB algorithm. In this paper, we theoretically analyze the maximum transmission delay of CSMA/CD, as one of the analysis methods by using the timing analysis of data link classes [3]. The maximum transmission delay of the existing Ethernet occurs in the following three kinds of situations; the maximum transmission delays occurs 1) after the transmitted frames collide 16 times, 2) occurs when the backoff time has the greatest value, $2 \mathrm{~min}$ (trial number 10), 3) occurs when $D_{D E T}$ has the greatest 
value - twice the value of the transmission delay between the source stations and the destinations. In these situations, the theoretical maximum collision delays are calculated as the equation (1).

$$
\begin{aligned}
D_{C} & =\sum_{k=1}^{16}\left(D_{D E T}+D_{J A M}+D_{B O \max }\right) \\
& =16\left(4 D_{P R O P}+D_{J A M}\right)+\sum_{k=1}^{16} 2^{\min (k, 10)} t_{s} \\
& =4,185,920 t_{b} \approx 418.6 \mathrm{~m} \mathrm{~s}
\end{aligned}
$$

where $D_{B O \max }$ : Maximum backoff time with BEB algorithm

$D_{J A M}:$ Jam transmission time

$D_{P R O P}$ : Propagation delay, $D_{D E T}$ : Collision detection time

From the above analysis, we can identify that the maximum transmission delay occurring in Ethernet is about $418.6 \mathrm{~m} \mathrm{~s}$. This result shows that the direct application to industrial network is not concise because if the traffic of Ethernet increases, the number of collisions and the following transmission delays drastically increase.

Theoretical maximum transmission delays of switched Ethernet occur when $N_{q}$ value is maximum which is determined by the network traffic and the quantity of switched buffer. If the network traffic is stable, and only the regular frames are generated, the value of $N_{q}$ will be maximized because the processing ability of switches per hour is big enough when the frames with the same destinations are generated simultaneously and stored in switches. The maximum transmission delay time $D_{Q}$ is like the equation (2).

$$
\begin{aligned}
D_{Q} & =\sum_{k=1}^{N_{q}}\left(D_{I F}+D_{T K}\right)=\sum_{k=1}^{N_{q}}\left(D_{I F}+\max \left(L_{k}+L_{h}, 576\right) t_{b}\right. \\
& =N_{q}\left(96+\max \left(K_{k}+432,576\right) t_{b}\right. \\
& =13 \times 672 \times t_{b}=873.6 \mu \mathrm{sec}
\end{aligned}
$$

where $D_{I F}$ : Interframe delay

$D_{T K}$ : Message length of $k^{\text {th }}$

$N_{q}$ : The number of frames stored in switches

$L_{k}$ : Data size of $k^{\text {th }}, L_{h}$ : Overhead of transmitted frames

According to equation (2), we know that if switched Ethernet is used, only a small transmission delay occurs. The reason for the result is that there is no collision in the switched Ethernet. The switched Ethernet satisfies the real-time requirements for the industrial networks, and it can be applied to railway signaling systems. 


\section{Network algorithm in railway signaling systems}

In this paper, we study the methods of developing switched Ethernet reliability to apply them to railway signaling systems in which the safety is critical. Switched Ethernet satisfies the real-time requirements, but the reliability can not be guaranteed when each component is broken down because system components are related with each other such as Network-Interface Card (NIC), switch HUB, and communication lines. To solve this problem, we propose the multiple architecture where each component is duplicated, and estimate its performance.

\subsection{Architecture of hardware redundancy}

\subsubsection{Switch HUB}

In Ethernet communications, the data cables are shared, and all nodes have their individual addresses, and the communications are also done by the addresses through switching HUBs. In the beginning stage of switching HUB, unavoidable collisions occurred as traffics gradually increasing because the HUB, which never buffer total packets, has just a destination addresses and takes the way to transmit. Most of the currently realized switching HUBs don't bring any collisions excluding queue delay in switches, because they have independent queues of more than two in every port, and store the whole data in the way of Store \& Forward, and then transmit the data after confirming destination addresses.

\subsubsection{MultiPort Network Interface Card for fault-tolerant characteristic}

Fault Tolerant is a system which is designed not to disturb system operations even in the case that some modules or components in the system have faults. The aim of the Fault Tolerant system is to continue operating the system regularly with no loss and destruction of data despite the occurrences of faults. The fault tolerance goes through the following three steps, these stages are proceeded and linked with running application software.

1. Fault Detection

Fault Detection is usually operated by Compare Logic constructed by hardware. When faults appear in systems, the decided modules or the systems come into the fault-condition. When the faults appear, OS analyzes every condition of hardware modules, and identifies which module makes faults.

2. Fault Diagnosis

The characteristics of faults are transient or hard. If the faults are hard, the modules of the systems are removed. If they are transient, that the self diagnoses result in no problem, the system first recognizes the fault as transient faults, and performs all operations.

3. Fault Recovery

Fault Recovery reconstructs systems by removing the modules which bring faults. One of the important functions, which keeps the fault tolerance, is to 
duplicate data more than twice and then finally keeps the fault tolerance. In other words, when some data are created from application programs, the fault recovery always stores the data in two memories, and then transmits the data. Even when a module has some faulty data, fault recovery can keep the fault tolerance giving no influence on other systems. In this paper, we embody the Redundancy system by using NIC which has four Modules.

\subsubsection{System architecture with fault tolerance}

As mentioned above, to construct a fault tolerant system, multiple communication ports, and communication lines are needed. And also module redundancy should be embodied using multiple NIC, and redundancy in switches should be realized by linking switch HUBs doubly. If only one switch is installed, obstacles of total systems occur when faults are made in switches even though the redundancy in modules is guaranteed. Linking one communication line to each one port is another caution to avoid, because there is enough scope to make errors in the communication lines. The following Fig. 1 is the system architecture which meets those requirements.

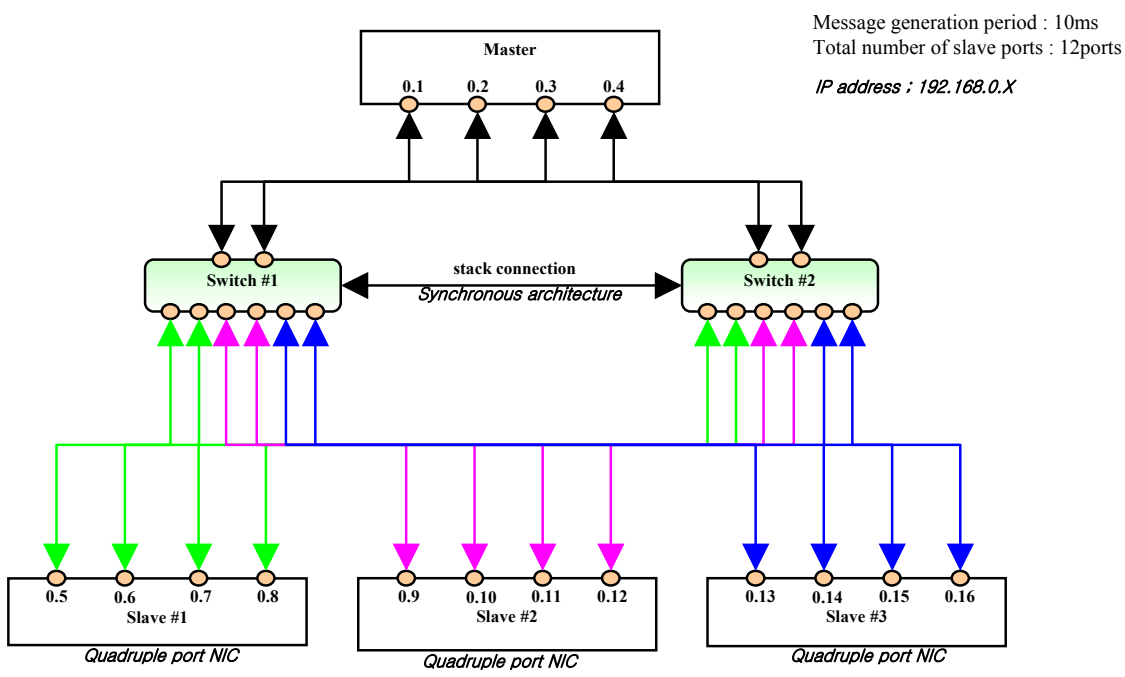

Figure 1: Overview of system architecture.

\subsection{The development of fault detection and operation algorithm for communication nodes}

In switched Ethernet, to double components, supporting software in application layer, $7^{\text {th }}$ level of OSI reference is also necessary, not only a system framework of hardware. At first, more than two bindings of network cards are supported in one station for the duplication of network cards, and sockets and software buffers more than twice used for communication are needed. 
The proposed system architecture is using four communication ports and four communication lines, and two switches, so the software for estimating the performance should be able to support system architecture as the above. Fig. 2 is the approximate program diagram of the software for full redundancy of the switched Ethernet. Explaining it approximately; a slave which generates messages continuously creates messages per $10 \mathrm{~ms}$, and the message-generating routine creates an event of message generation to a slave message-sending routine when messages are generated. The slave message-sending routine which receives the event is constructed by the way of transmitting messages through individually bounding ports. The master that receives the messages plays a role to carry perfect data to application levels after copying the data entered individual received buffers and bring to voting routines, and comparing the data.

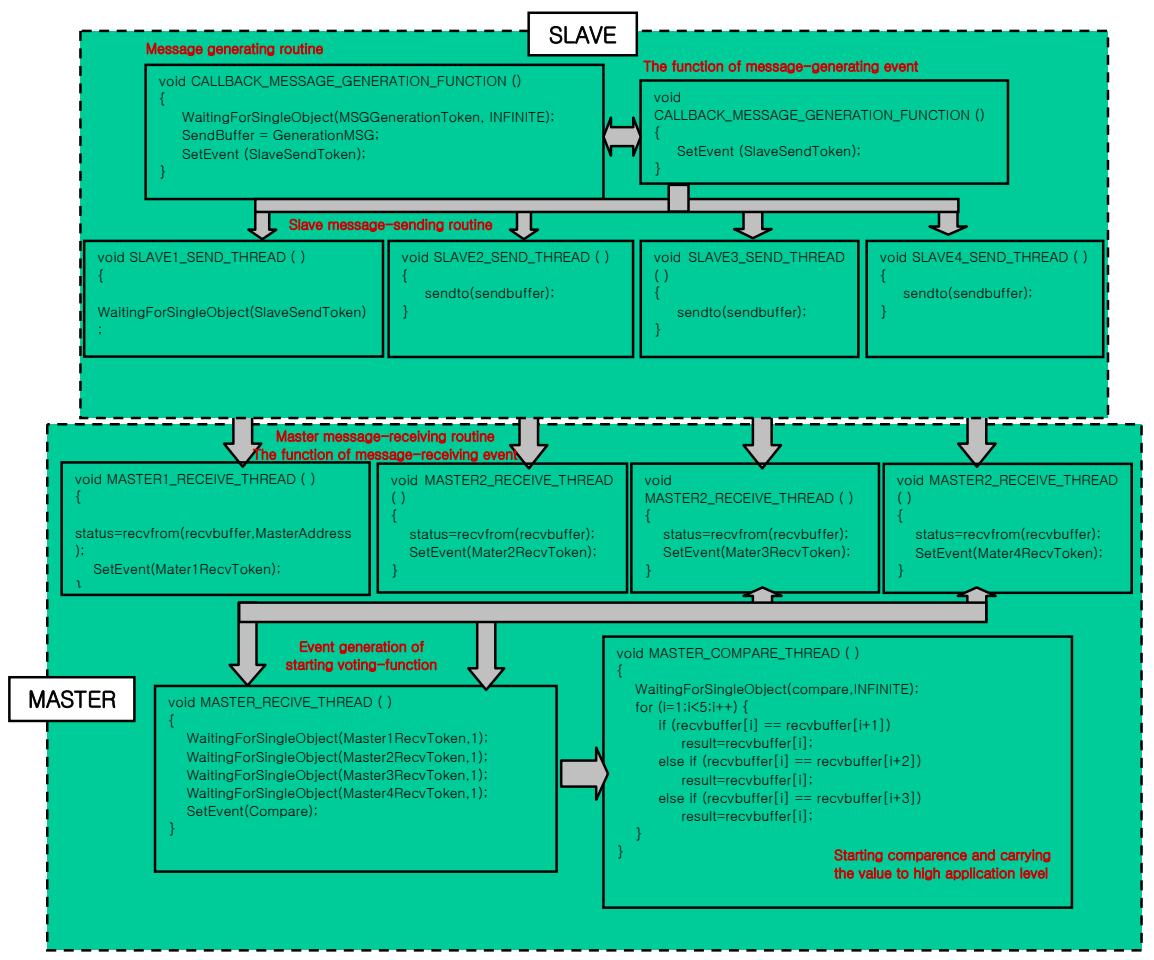

Figure 2: Test software configuration of proposed Ethernet architecture.

\section{Conclusion}

Railway signaling systems should necessarily guarantee reliability between the signaling systems because they are linked with other controlling equipment and construct bigger signal controlling systems, performing each function. The links of signaling systems has some problems with maintenance and repairs because the way of linking is of the point-to-point communication system. 
The safety-critical systems, such as railway signaling systems, signify the reliability with emphasizing transmission delay. For these necessities, fault tolerance should be realized in the environment which requires high reliability when applying network like cars, automobiles, aviation vehicles. In this paper, we propose the reliable switched Ethernet architecture with redundancy systems and analyse the performing efficiency by using NIC with four modules.

We verified the proposed structures by experiments on bus redundancy for communication line reliability, module redundancy for confirming communication module reliability, and switch redundancy for switch HUB reliability. All error-recovery rates are $100 \%$ - all in the situational cases when communication circuits are cut during the communication between slaves and masters, when the uses of modules are stopped in the same conditions, and when the power supplies to switches are cut. The fault detection time is also less than $1 \mathrm{~ms}$. For these reasons, we consider that the proposed systems show the reliability enough to make the systems applied to railway signaling systems.

\section{References}

[1] Hideo Nakamura, 'Development of Computerized Interlocking SMILENET', RTRI Report, 1986.

[2] Nishida Shigenobu and etc., 'Replacement of Tokaido Shinkansen Signalling System Providing Advanced Train Controls in the 21st Century', KYOSAN CIRCULAR, Vol. 53, No. 2, 2002.

[3] Kyung Chang Lee, Suk Lee, 'Performance Evaluation of Switched Ethernet for Real-time Industrial Communications', Computer Standards \& Interfaces, Vol. 24, No. 5, pp. 411-423, Nov. 2002.

[4] IEEE standard 802.3, Part 3: Carrier sense multiple access with collision detection $(\mathrm{CSMA} / \mathrm{CD})$ access method and physical layer specifications, 2000 . 